\title{
Interpolation Error Estimates for the Reduced Hsieh-Clough-Tocher Triangle
}

\author{
By Philippe G. Ciarlet
}

Abstract. We study the unisolvence and interpolation properties of the reduced Hsieh-Clough-Tocher triangle. This finite element of class $C^{1}$, which has only nine degrees of freedom, can be used in the numerical approximation of plate problems.

Introduction; Main Notation. The space $\mathbf{R}^{n}$ is equipped with the Euclidean norm $|\cdot|$ and inner-product $\langle\cdot, \cdot\rangle$. If $B$ is a subset of $\mathbf{R}^{n}$, we let

$h_{B}=$ diameter of $B$,

$\rho_{B}=\sup \left\{h_{B} ; B\right.$ is a ball contained in $\left.B\right\}$, meas $(B)=\int_{B} d x$ (assuming $B$ to be measurable), $\left.v\right|_{B}=$ restriction of the function $v$ to the set $B$,

$$
P_{k}(B)=\left\{\left.p\right|_{B} ; p \in P_{k}\left(\mathbf{R}^{n}\right)\right\}, \quad k \in \mathbf{N},
$$

where $P_{k}\left(\mathbf{R}^{n}\right)$ denotes the space formed by all polynomials of degree $\leqslant k$ in $n$ variables.

Given a multi-index $\alpha=\left(\alpha_{1}, \alpha_{2}, \ldots, \alpha_{n}\right) \in \mathbf{N}^{n}$ with length $|\alpha|=\sum_{i=1}^{n} \alpha_{i}$, we use the usual notation $\partial^{\alpha} v(a)$ for the partial derivatives of order $|\alpha|$ of a function $v$ at a point $a \in \mathbf{R}^{n}$, while the Fréchet derivatives are denoted $D^{m} v(a)$, or simply $D v(a)$ if $m=1$. We write for brevity

$$
D^{m} v(a)\left(\xi_{1}, \xi_{2}, \ldots, \xi_{m}\right)= \begin{cases}D^{m} v(a) \xi^{m} & \text { if } \xi_{1}=\xi_{2}=\cdots=\xi_{m}=\xi, \\ D^{m} v(a)\left(\xi^{m-1}, \xi_{m}\right) & \text { if } \xi_{1}=\xi_{2}=\cdots=\xi_{m-1}=\xi .\end{cases}
$$

Following [3] , [4], let us recall some general definitions pertaining to finite elements: A finite element in $\mathbf{R}^{n}$ is a triple $(K, P, \Sigma)$, where:

(i) $K$ is a subset of $\mathbf{R}^{n}$ with a nonempty interior $\stackrel{\circ}{K}$ and a Lipschitz-continuous boundary in the sense of Nečas [7],

(ii) $P$ is a vector space of finite dimension $N$, whose elements are real-valued functions defined over the set $K$,

(iii) $\Sigma$ is a set of $N$ linear forms $\phi_{i}, 1 \leqslant i \leqslant N$, defined over the space $P$, and the set $\Sigma$ is $P$-unisolvent in the following sense: Given arbitrary real numbers $\alpha_{i}, 1 \leqslant$ $i \leqslant N$, there exists one and only one function $p \in P$ which satisfies

$$
\phi_{i}(p)=\alpha_{i}, \quad 1 \leqslant i \leqslant N .
$$

The forms $\phi_{i}$ are called the degrees of freedom of the finite element. The basis

Received May 5, 1977.

AMS (MOS) subiect classifications (1970). Primary 41A25, 65D05, 65N 15, 65N30.

Copyright $\odot$ 1978, American Mathematical Society 
function $r_{j}$ associated with the degree of freedom $\phi_{j}$ is defined by the relations

$$
r_{j} \in P \quad \text { and } \quad \phi_{i}\left(r_{j}\right)=\delta_{i j}, \quad 1 \leqslant i, j \leqslant N .
$$

Notice that the functions $r_{j}$ form indeed a basis in the space $P$ since the identity

$$
\forall p \in P, \quad p=\sum_{j=1}^{N} \phi_{j}(p) r_{j}
$$

holds.

Given a function $v$ defined over the set $K$, the P-interpolant $\Pi v$ of the function $v$ is defined through the relations

$$
\Pi v \in P \quad \text { and } \quad \phi_{i}(\Pi v)=\phi_{i}(v), \quad 1 \leqslant i \leqslant N .
$$

Equivalently, we have

$$
\Pi v=\sum_{j=1}^{M} \phi_{j}(v) r_{j} .
$$

Of course, the above definition makes sense only if the function $v$ is smooth enough so that the degrees of freedom $\phi_{j}(v)$ are well defined. For finite elements for which all the degrees of freedom are of the form $\partial^{\alpha} v(a)$ (as is the case in this paper), we shall require, for definiteness, that the function $v$ be $s$ times continuously differentiable over the set $K$, where $s$ is the maximal order of partial derivatives found in the set $\Sigma$. In other words, for such finite elements, we can define a P-interpolation operator

$$
\Pi: \operatorname{dom} \Pi=C^{s}(K) \rightarrow P .
$$

A reduced Hsieh-Clough-Tocher triangle is a triple $(K, P, \Sigma)$ where the data $K, P$ and $\Sigma$ are defined as follows.

(i) The set $K$ is a triangle, with vertices $a_{1}, a_{2}, a_{3}$.

(ii) Let $a$ be any point in the interior of the triangle $K$. Denoting (cf. Figure 1) by $K_{i}$ the triangle with vertices $a, a_{i+1}, a_{i+2}$ (the indices are counted modulo 3

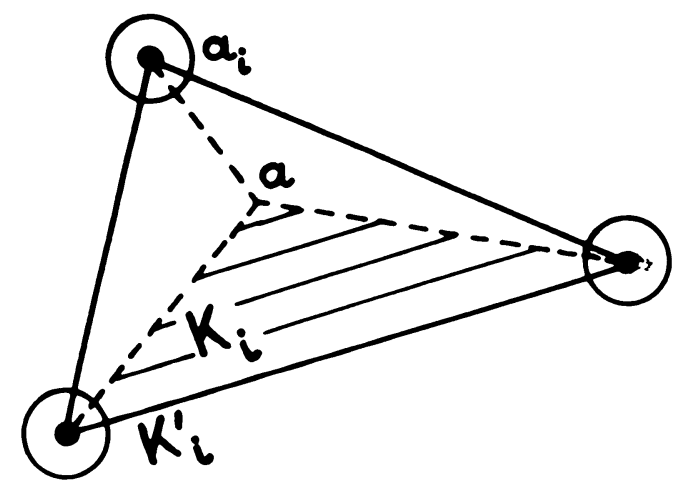

FIGURE 1

whenever necessary), and by $K_{i}^{\prime}$ the side opposite to the vertex $a_{i}$, the space $P$ is given by

$$
P=\left\{p \in \mathcal{C}^{1}(K) ;\left.p\right|_{K_{i}} \in P_{3}\left(K_{i}\right),\left.\partial_{\nu} p\right|_{K_{i}^{\prime}} \in P_{1}\left(K_{i}^{\prime}\right), 1 \leqslant i \leqslant 3\right\}
$$


where $\left.\partial_{\nu} p\right|_{K_{i}^{\prime}}$ is the (outer) normal derivative of the function $\left.p\right|_{K_{i}}$ along the side $K_{i}^{\prime}$.

(iii) The set of degrees of freedom is

$$
\Sigma=\left\{\partial^{\alpha} p\left(a_{i}\right), 1 \leqslant i \leqslant 3,|\alpha| \leqslant 1\right\}
$$

This finite element is derived from the standard Hsieh-Clough-Tocher triangle by a device common to triangular finite elements of class $C^{1}$, which consists in constraining the normal derivative on the exterior sides to be polynomials of lower degree. The Hsieh-Clough-Tocher triangle (which is described later in this paper, cf. (6)) is narned after Clough and Tocher [6], and also after Hsieh, who was the first to conceive in 1962 the idea of matching three polynomials so as to get a finite element of class $C^{1}$. We recall that a finite element is said to be of class $C^{1}$ if, whenever it is assembled in a triangulation, the resulting functions and their partial derivatives of order one are continuous across the sides common to adjacent triangles. Since this is the case of the reduced Hsieh-Clough-Tocher triangle, and since its interpolation properties make it amenable for solving fourth-order problems (cf. (5)), this finite element is optimal in the sense that it has the lowest possible number of degrees of freedom, that is, nine, compatible with a cubic variation of the function and a linear variation of the normal derivative along the exterior sides. In particular, we obtain a finite element of class $C^{1}$ for which the dimension of the space $P$ is quite small, compared to more traditional triangular finite elements of class $C^{1}$ where only "pure" polynomials are used. We recall that for such elements, Ženíšek [11] has shown that the dimension of the space $P$ is at least 18. Notice, however, that this decrease in the dimension, an obvious computational advantage as regards the dimension of the resulting linear system, is obtained at the expense of an increased complexity in the structure of the space $P$. As expected, the basis functions are harder to compute explicitly.

After we have shown (Theorem 1) that the set $\Sigma$ is $P$-unisolvent, with $P$ and $\Sigma$ given as in (1) and (2), we turn to the main object of this paper, which is to estimate the interpolation errors $|v-\Pi v|_{m, K}$, where the standard notation

$$
|w|_{m, A}=\left(\int_{A} \sum_{|\alpha|=m}\left|\partial^{\alpha} v\right|^{2} d x\right)^{1 / 2},
$$

is used, and where, according to the general definition given above, the $P$-interpolant $\Pi v$ is uniquely determined by the conditions

$$
\Pi v \in P \quad \text { and } \quad \partial^{\alpha}(\Pi v)\left(a_{i}\right)=\partial^{\alpha} v\left(a_{i}\right), \quad 1 \leqslant i \leqslant 3,|\alpha| \leqslant 1 .
$$

We are then able to show that (Theorem 2), given a family of reduced Hsieh-CloughTocher triangles which is regular in a sense to be defined below, and given a function $v \in H^{3}(K) \subset C^{1}(K)=$ dom $\Pi$, one has

$$
|v-\Pi v|_{m, K}=O\left(h_{K}^{3-m}\right), \quad m=0,1,2,
$$

i.e., the order of convergence is the same as one would expect from the inclusion $P_{2}(K) \subset P_{K}$ for an affine family in the sense of [5] (this is thus another instance of an almost-affine family of finite elements, according to the terminology of $[4$, Chap- 
ter 6]). It appeared, however, that the standard techniques for getting interpolation error estimates for finite elements of class $C^{1}$ (cf. Bramble and Zlámal [1], the author [3] , [4], Raviart [9], Ženíšek [10], Zlámal [13]) did not directly apply to this element, and this observation led to the present paper.

Let us then assume that we are using this finite element for solving a fourthorder problem (such as a plate problem) posed over some open set $\Omega \subset \mathbf{R}^{2}$. If we let $u$ and $u_{h}$ denote, respectively, the exact and approximate solution, then we get from (4),

$$
\left\|u-u_{h}\right\|_{H^{2}(\Omega)} \leqslant C h|u|_{3, \Omega},
$$

where $h$ denotes the greatest diameters of the triangles found in the finite element space where the discrete solution $u_{h}$ is found. Notice that the above error estimate requires that the solution $u$ be in the space $H^{3}(\Omega)$, but this is a mild regularity assumption, satisfied if $\bar{\Omega}$ is a convex polygon for a plate problem.

The reader interested in finite element methods for fourth-order problems in general may consult Zienkiewicz [12, Chapter 10] for a discussion from an engineering viewpoint, while a fairly complete description and a study of their convergence properties are given in [3, Sections 13 and 14] and [4, Chapters 6 and 7].

\section{Unisolvence.}

Theorem 1. The set $\Sigma$ of (2) is P-unisolvent, the space $P$ being defined as in (1).

Proof. Since the number of degrees of freedom is equal to the dimension of the space $P$, it suffices to prove that a function $p$ which satisfies

$$
p \in P \quad \text { and } \quad \partial^{\alpha} p\left(a_{i}\right)=0, \quad 1 \leqslant i \leqslant 3,|\alpha| \leqslant 1,
$$

is identically zero. The conjunction of these relations and the relations

$$
\left.\partial_{\nu} p\right|_{K_{i}^{\prime}} \in P_{1}\left(K_{i}^{\prime}\right) \text { and }\left.\partial_{\nu} p\right|_{K_{i}^{\prime}}\left(a_{i+1}\right)=\left.\partial_{\nu} p\right|_{K_{i}^{\prime}}\left(a_{i+2}\right)=0 \text {, }
$$

obtained from the definition of the space $P$, implies that

$$
\left.\partial_{\nu} p\right|_{K_{i}^{\prime}}=0, \quad 1 \leqslant i \leqslant 3 .
$$

The unisolvence is then a consequence of the unisolvence established in [2] (see also Percell [8] for another proof) for the Hsieh-Clough-Tocher triangle: This finite element is a triple $\left(K, P^{*}, \Sigma^{*}\right)$, with

$$
\left\{\begin{array}{l}
K=\text { a triangle subdivided as in Figure } 1 \\
P^{*}=\left\{p \in C^{1}(K),\left.p\right|_{K_{i}} \in P_{3}\left(K_{i}\right), 1 \leqslant i \leqslant 3\right\} \\
\Sigma^{*}=\left\{\partial^{\alpha} p\left(a_{i}\right),|\alpha| \leqslant 1, \partial_{\nu} p\left(b_{i}\right), 1 \leqslant i \leqslant 3\right\}
\end{array}\right.
$$

where $\partial_{\nu} p\left(b_{i}\right)$ denotes the normal derivative at the midpoint $b_{i}$ of the side $K_{i}^{\prime}$.

Interpolation Error Estimates. Our first objective is to appropriately describe a family of reduced Hsieh-Clough-Tocher triangles. Let $\hat{K}$ be a fixed triangle with vertices $\hat{a}_{i}$. Given an arbitrary triangle $K$ with vertices $a_{i, K}$ (cf. Figure 2), we let $F_{K}$ 


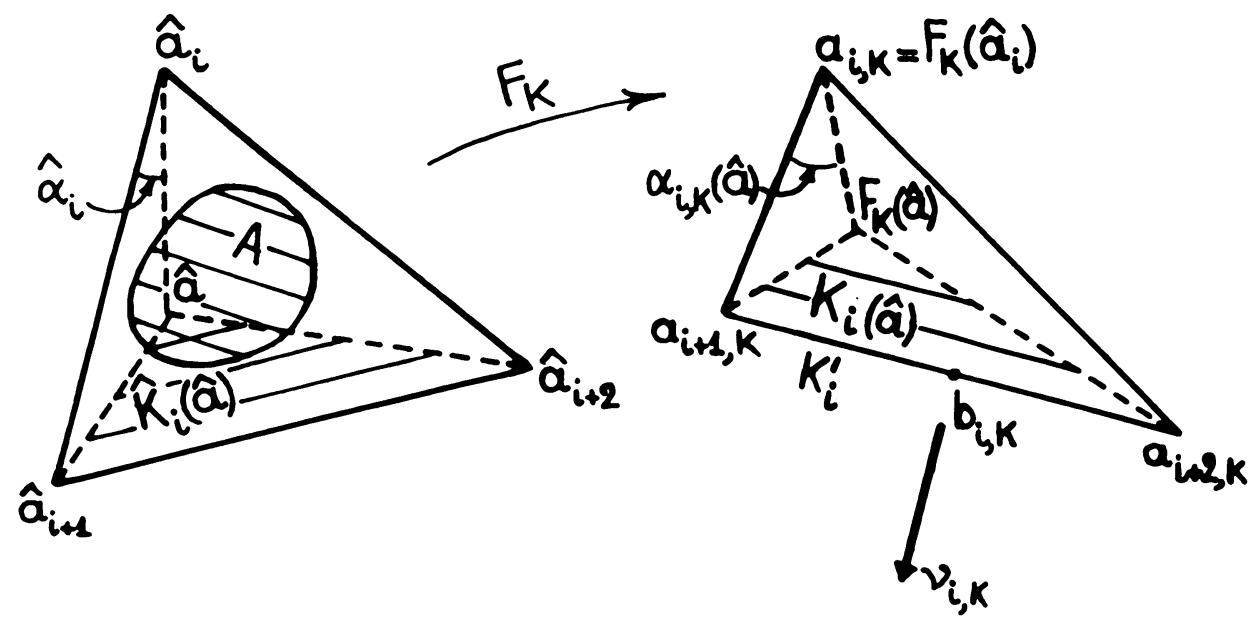

Figure 2

denote the affine mapping from $\mathbf{R}^{2}$ into $\mathbf{R}^{2}$ uniquely determined by the conditions

$$
a_{i, K}=F_{K}\left(\dot{a}_{i}\right), \quad 1 \leqslant i \leqslant 3 .
$$

Given a point $\hat{a}$ interior to the triangle $\hat{K}$, let, for $i=1,2,3, K_{i}(\hat{a})$ denote the triangle with vertices $F_{K}(\hat{a}), a_{i+1, K}, a_{i+2, K}$ (cf. Figure 2 ). Then we need the following definitions:

$\left(K, P_{K}(\hat{a}), \Sigma_{K}\right)=$ reduced Hsieh-Clough-Tocher triangle constructed on the triangle $K$ subdivided into the three triangles $K_{i}(\hat{a}), 1 \leqslant i \leqslant 3$,

$$
\begin{aligned}
& \Pi_{K}(\hat{a})=P_{K}(\hat{a}) \text {-interpolation operator associated } \\
& \text { with the finite element }\left(K, P_{K}(\hat{a}), \Sigma_{K}\right)
\end{aligned}
$$

where $b_{i, K}$ is the midpoint of the side opposite to the vertex $a_{i, K}$,

$$
\Lambda_{K}(\hat{a})=Q_{K}(\hat{a}) \text {-interpolation operator associated with the finite }
$$
element $\left(K, Q_{K}(\hat{a}), \Xi_{K}(\hat{a})\right)$ (the proof of the $Q_{K}(\hat{a})$-unisolvence of the set $\Xi(\hat{a})$ is the same as for the Hsieh-Clough-Tocher triangle).

Following [2], we are then naturally led to define a regular family of reduced Hsieh-Clough-Tocher triangles as a family

$$
\left(K, P_{K}(\hat{a}), \Sigma_{K}\right)_{K \in K, \hat{a} \in A}
$$

for which:

(i) There exists a constant $\sigma$ independent of $K \in K$ such that

$$
\forall K \in K, \quad h_{K} \leqslant \sigma \rho_{K},
$$

(ii) Zero is the only point adherent to the set $\left\{h_{K} \in \mathbf{R} ; K \in K\right\}$, 

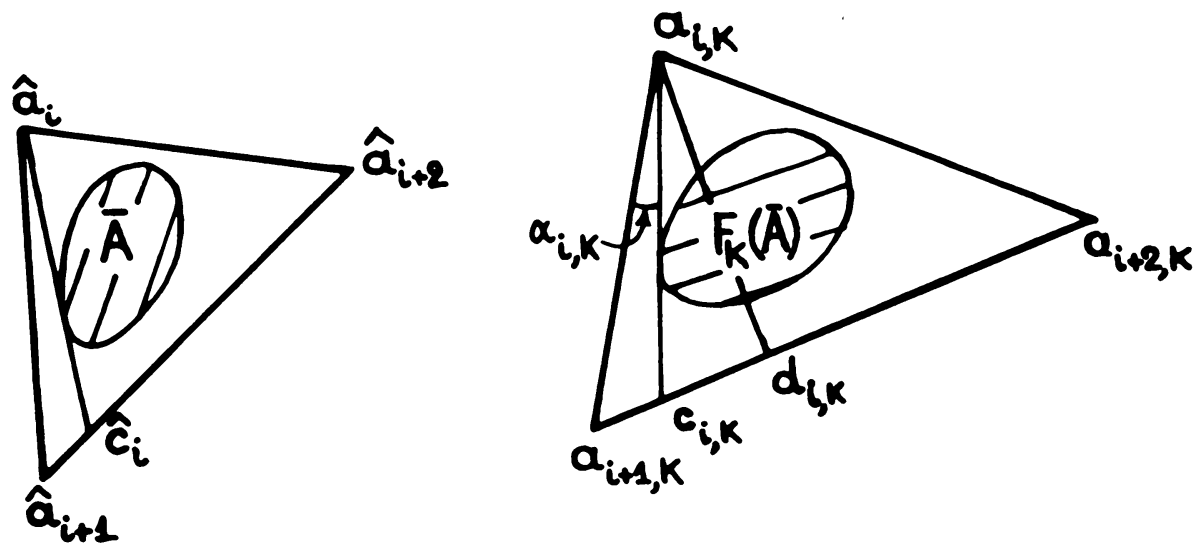

FIGURE 3

(iii) The set $\bar{A}$ is a compact subset of the interior of the triangle $\hat{K}$ (cf. Figure 2 ). To begin with, we establish a result of a purely geometrical nature.

LEMmA 1. Let there be given a regular family of reduced Hsieh-Clough-Tocher triangles. Then there exists a constant $v$ such that

$$
\forall K \in K, \quad \forall \hat{a} \in A, \quad h_{K} \leqslant \nu \rho_{K_{i}(\hat{a})}, \quad 1 \leqslant i \leqslant 3 .
$$

Proof. It suffices to show that an angle such as $\alpha_{i, K}(\hat{a})$ (cf. Figure 2) is bounded below by a strictly positive constant independently of $K \in K$ and $\hat{a} \in A$. Equivalently, we shall show that

$$
\inf _{K \in K} \alpha_{i, K}>0
$$

where, for each $K \in K$ (cf. Figure 3),

$$
\alpha_{i, K}=\inf _{a \in F_{K}(\bar{A})}\left\{\operatorname{angle}\left(a-a_{i, K}, a_{i+1, K}-a_{i, K}\right)\right\} .
$$

Let us assume the contrary. With the notation of Figure 3 , the ratio $\left|c_{i, K}-a_{i+1, K}\right|\left(\left|a_{i+2, K}-a_{i+1, K}\right|\right)^{-1}$ is a constant independent of $K \in K$, since it is equal to the ratio $\left|\hat{c}_{i}-\hat{a}_{i+1}\right|\left(\left|\hat{a}_{i+2}-\hat{a}_{i+1}\right|\right)^{-1}$. Therefore, the equality $\inf _{K \in K} \alpha_{i, K}=0$ would imply that the ratio $\left|a_{i, K}-a_{i+1, K}\right|\left(\left|a_{i+2, K}-a_{i+1, K}\right|\right)^{-1}$ approaches infinity or that the ratio $\left|d_{i, K}-a_{i, K}\right|\left(\left|a_{i+2, K}-a_{i+1, K}\right|\right)^{-1}$ approaches zero, where $\left(d_{i, K}-a_{i, K}\right)$ is the height issued from $a_{i, K}$ (cf. Figure 3 ). But neither implication is compatible with assumption (12); and, therefore, we have reached a contradiction.

We are then in a position to prove our main result.

THEOREM 2. Given a regular family of reduced Hsieh-Clough-Tocher triangles, there exists a constant $C$ such that

$$
\begin{aligned}
\forall K \in K, \quad \forall \hat{a} \in A, \quad \forall v \in H^{3}(K), \\
\quad\left|v-\Pi_{K}(\hat{a}) v\right|_{m, K} \leqslant C h_{K}^{3-m}|v|_{3, K}, \quad m=0,1,2 .
\end{aligned}
$$

Proof. There are two reasons that prevent us from using the "affine" interpolation theory: 
(a) The points $\hat{a}$ may vary inside $\hat{K}$ : This possibility will require the use of a compactness argument, as in [2].

(b) Given a subfamily corresponding to a fixed point $\hat{a}$, the spaces $P_{K}(\hat{a})$ are not in an affine correspondence (since the sets $\Sigma_{K}$ are in such a correspondence, we are therefore in a situation opposite to that corresponding to the Hsieh-Clough-Tocher triangle; cf. [2]).

In what follows, the letter $C$ stands for any constant (not necessarily the same in its various occurrences) independent of $K \in K, \hat{a} \in A$, and of the functions occurring in the inequality where $C$ appears.

The proof is subdivided into several steps.

(i) We first notice that the inclusion

$$
H^{3}(K) \subset C^{1}(K)=\operatorname{dom} \Pi_{K}(\hat{a})=\operatorname{dom} \Lambda_{K}(\hat{a})
$$

holds (cf. definitions (8) and (11)). Next the same argument as in [2, Eq. (3.4)] or [4, Eq. (6.1-31)] shows that there exists a constant $C$ such that

$$
\begin{aligned}
\forall K \in K, \quad \forall \hat{a} \in A, \quad \forall v \in H^{3}(K), \\
\quad\left|v-\Lambda_{K}(\hat{a}) v\right|_{m, K} \leqslant C h_{K}^{3-m}|v|_{3, K}, \quad m=0,1,2 .
\end{aligned}
$$

(ii) In view of estimating the seminorms $\left|\Lambda_{K}(\hat{a}) v-\Pi_{K}(\hat{a}) v\right|_{m, K}$, let us expand the difference $\left(\Lambda_{K}(\hat{a}) v-\Pi_{K}(\hat{a}) v\right)$ over the basis functions of the finite element $\left(K, Q_{K}(\hat{a}), \Xi_{K}(\hat{a})\right)$ (cf. definitions (9), (10)). This is indeed possible since the inclusion $P_{K}(\hat{a}) \subset Q_{K}(\hat{a})$ holds. Denoting by $r_{i, K}(\hat{a})$ the basis function associated with the degree of freedom $D p\left(b_{i, K}\right)\left(F_{K}(\hat{a})-b_{i, K}\right)$ and using the respective interpolation properties of the functions $\Lambda_{K}(\hat{a}) v$ and $\Pi_{K}(\hat{a}) v$, we obtain:

$$
\Lambda_{K}(\hat{a})-\Pi_{K}(\hat{a}) v=\sum_{i=1}^{3}\left\{D\left(\Lambda_{K}(\hat{a}) v-\Pi_{K}(\hat{a}) v\right)\left(b_{i, K}\right)\left(F_{K}(\hat{a})-b_{i, K}\right)\right\} r_{i, K}(\hat{a}) .
$$

Let us introduce the unit vector $v_{i, K}$ normal to the side $K_{i}^{\prime}$ opposite to the vertex $a_{i, K}$, as indicated in Figure 2. The equality of the functions $\Lambda_{K}(\hat{a}) v$ and $\Pi_{K}(\hat{a}) v$ along the side $K_{i}^{\prime}$ implies that

$$
\begin{aligned}
& D\left(\Lambda_{K}(\hat{a}) v-\Pi_{K}(\hat{a}) v\right)\left(b_{i, K}\right)\left(F_{K}(\hat{a})-b_{i, K}\right) \\
& \quad=\partial_{\nu}\left(\Lambda_{K}(\hat{a}) v-\Pi_{K}(\hat{a}) v\right)\left(b_{i, K}\right)\left\langle F_{K}(\hat{a})-b_{i, K}, v_{i, K}\right\rangle,
\end{aligned}
$$

and thus we get from (16):

$$
\left|\Lambda_{K}(\hat{a}) v-\Pi_{K}(\hat{a}) v\right|_{m, K} \leqslant h_{K} \sum_{i=1}^{3}\left|\partial_{\nu}\left(\Lambda_{K}(\hat{a}) v-\Pi_{K}(\hat{a}) v\right)\left(b_{i, K}\right)\right|\left|r_{i, K}(\hat{a})\right|_{m, K}
$$

(iii) Let us next transform the expressions

$$
\partial_{\nu}\left(\Lambda_{K}(\hat{a}) v-\Pi_{K}(\hat{a}) v\right)\left(b_{i, K}\right)
$$

To do this, we shall use the following result: Given a (smooth enough) function $w$ whose restriction to the side $K_{i}^{\prime}$ belongs to the space $P_{2}\left(K_{i}^{\prime}\right)$, we have

$$
w\left(b_{i, K}\right)=\frac{1}{2}\left\{w\left(a_{i+1, K}\right)+w\left(a_{i+2, K}\right)\right\}-\frac{1}{8} D^{2} w\left(b_{i, K}\right)\left(a_{i+2, K}-a_{i+1, K}\right)^{2}
$$


Applying identity (18) to the functions

$$
\partial_{\nu} \Lambda_{K}(\hat{a}) v \in P_{2}\left(K_{i}^{\prime}\right) \quad \text { and } \quad \partial_{\nu} \Pi_{K}(\hat{a}) v \in P_{1}\left(K_{i}^{\prime}\right)
$$

and taking into account the respective interpolation properties of the functions $\Lambda_{K}(\hat{a}) v$ and $\Pi_{K}(\hat{a}) v$ at the points $a_{i+1, K}$ and $a_{i+2, K}$, we obtain

$$
\partial_{\nu}\left(\Lambda_{K}(\hat{a}) v-\Pi_{K}(\hat{a}) v\right)\left(b_{i, K}\right)=-\frac{1}{8} D^{3}\left(\Lambda_{K}(\hat{a}) v\right)\left(b_{i, K}\right)\left(\left(a_{i+2, K}-a_{i+1, K}\right)^{2}, v_{i, K}\right) .
$$

Therefore,

$$
\left|\partial_{\nu}\left(\Lambda_{K}(\hat{a}) v-\Pi_{K}(\hat{a}) v\right)\left(b_{i, K}\right)\right| \leqslant C h_{K}^{2}\left|\Lambda_{K}(\hat{a}) v\right|_{3, \infty, K_{i}(\hat{a})}, \quad 1 \leqslant i \leqslant 3,
$$

where

$$
|w|_{m, \infty, B}=\max _{|\alpha|=m}\left\|\partial^{\alpha} w\right\|_{L^{\infty}(B)} .
$$

Notice in passing that in the right-hand side of inequality (19), we cannot use the seminorm $|\cdot|_{3, \infty, K}$, since the space $P_{K}(\hat{a})$ is not contained in the space $W^{3, \infty}(K)$ (it is only contained in the space $W^{2, \infty}(K)$ ).

(iv) We now estimate the seminorms $\left|\Lambda_{K}(\hat{a}) v\right|_{3, \infty, K_{i}(\hat{a})}$ in terms of the semi-

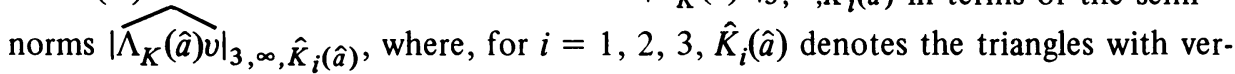
tices $\hat{a}, \hat{a}_{i+1}, \hat{a}_{i+2}$ (cf. Figure 2), and where, given a function $w: K \rightarrow \mathbf{R}$, we define the function $\hat{w}=\hat{K} \rightarrow \mathbf{R}$ by letting

$$
\forall \hat{x} \in \hat{K}, \quad \hat{w}(\hat{x})=w\left(F_{K}(\hat{x})\right) .
$$

Using the standard formulas for transforming Sobolev seminorms (see e.g. [3, Section 6] or [4, Theorems 3.1-2 and 3.1-3]), we get

$$
\left|\Lambda_{K}(\hat{a}) v\right|_{3, \infty, K_{i}(\hat{a})} \leqslant C\left(h_{\hat{K}_{i}(\hat{a})}\right)^{3}\left(\rho_{K_{i}(\hat{a})}\right)^{-3} \mid \widehat{\left.\Lambda_{K}(\hat{a}) v\right|_{3, \infty, \hat{K}_{i}(\hat{a})}}
$$

Combining this inequality with inequality (13) of Lemma 1 , we obtain

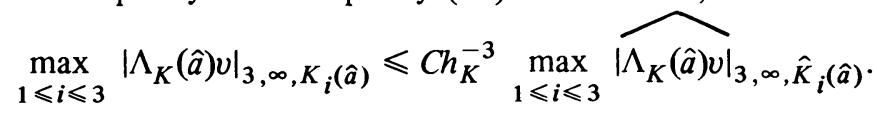

(v) Given an arbitrary function $\hat{p}$ in the space $Q_{\hat{K}}(\hat{a})$ and a multi-index $\alpha$ with $|\alpha|=3$, we have

$$
\left|\partial^{\alpha} \hat{p}\right|_{0, \infty, \hat{K}_{i}(\hat{a})}=\left\{\operatorname{meas} \hat{K}_{i}(\hat{a})\right\}^{-1 / 2}\left|\partial^{\alpha} \hat{p}\right|_{0, \hat{K}_{i}(\hat{a})},
$$

since $\partial^{\alpha} p \hat{K}_{i}(\hat{a})$ is a constant function. Consequently,

$$
\begin{aligned}
\max _{1 \leqslant i \leqslant 3}|\hat{p}|_{3, \infty, \hat{K}_{i}(\hat{a})} & \leqslant \max _{1 \leqslant i \leqslant 3}\left\{\operatorname{meas} \hat{K}_{i}(\hat{a})\right\}^{-1 / 2}|\hat{p}|_{3, \hat{K}_{i}(\hat{a})} \\
& \leqslant C\left(\sum_{i=1}^{3}|\hat{p}|_{3, \hat{K}_{i}(\hat{a})}^{2}\right)^{1 / 2}
\end{aligned}
$$

since

$$
\inf _{\hat{a} \in A} \operatorname{meas} \hat{K}_{i}(\hat{a})>0, \quad 1 \leqslant i \leqslant 3
$$


(to prove this last property, observe that an angle such as $\hat{\alpha}_{i}$ (cf. Figure 2) is $>0$ ). Applying this to the function $\hat{p}=\widehat{\Lambda_{K}(\hat{a}) v}$, we obtain

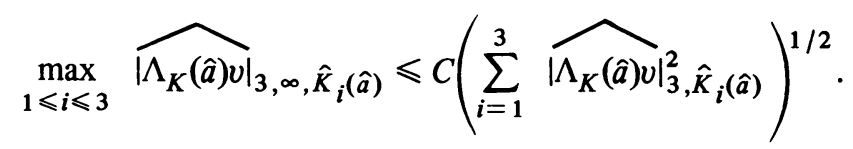

(vi) Using the correspondence (20), we notice that

$$
\widehat{\Lambda_{K}(\hat{a}) v}=\hat{\Lambda}(\hat{a}) \hat{v}
$$

where $\hat{\Lambda}(\hat{a})$ is the $Q_{\dot{K}}(\hat{a})$-interpolation operator associated with the finite element $\left(\hat{K}, Q_{\hat{K}}(\hat{a}), \Xi_{\hat{K}}(\hat{a})\right)$. Because this interpolation operator leaves the space $P_{2}(\hat{K})$ invariant, an easy adaptation of the compactness argument used in [2] or in [4, Theorem 6.1-3] shows that

$$
\sup _{\hat{a} \in A}\left(\sum_{i=1}^{3}|\hat{v}-\hat{\Lambda}(\hat{a}) \hat{v}|_{3, \hat{K}_{i}(\hat{a})}\right)^{1 / 2} \leqslant C|\hat{v}|_{3, \hat{K}}
$$

By combining (23), (24) and the triangular inequality, we obtain

$$
\left(\sum_{i=1}^{3}\left|\widehat{\Lambda_{K}(\hat{a}) v \mid}\right|_{3, \hat{K}_{i}(\hat{a})}\right)^{1 / 2} \leqslant C|\hat{v}|_{3, \hat{K}} .
$$

(vii) Using again the inequalities for transforming Sobolev seminorms, we have

$$
|\hat{v}|_{3, \hat{K}} \leqslant C h_{K}^{2}|v|_{3, K}
$$

(viii) Another compactness argument, as in [2] or [4, Theorem 6.1-3] shows that

and consequently,

$$
\sup _{\hat{a} \in A}\left|\widehat{r}_{i, K}(\hat{a})\right|_{m, \hat{K}}<\infty, \quad m=0,1,2,
$$

$$
\sup _{\hat{a} \in A}\left|r_{i, K}(\widehat{a})\right|_{m, K} \leqslant C h_{K}^{1-m}, \quad m=0,1,2 .
$$

(ix) To finish the proof, it suffices to combine inequalities (15), (17), (19), (21), (22), (25), (26), (27), established in the previous steps.

Remarks. (i) The same type of proof applies to the 18-degree of freedom triangle, with a double simplification in that the corresponding space $P$ consists of pure polynomials (there are no assembled subtriangles), and there is no variable point such as $a$. Thus, for this finite element, the present approach provides an alternate proof to that given by Bramble and Zlámal [1].

(ii) The interpolation error estimates (14) of Theorem 2 can be generalized. In particular, one can establish under the same assumptions that

$$
\left(\sum_{i=1}^{3}\left|v-\Pi_{K}(\hat{a}) v\right|_{3, K_{i}(\hat{a})}^{2}\right)^{1 / 2} \leqslant C|v|_{3, K}
$$

(we cannot let $m=3$ in (14) because the space $P_{K}(\hat{a})$ is not contained in the space $H^{3}(K)$; it is only contained in the space $\left.H^{2}(K)\right)$. Another generalization would consist, 
as in [4, Chapter 6], in considering more general Sobolev seminorms in both sides of the interpolation error estimates.

Analyse Numérique, Tour 55-65

Université Pierre et Marie Curie

4 Place Jussieu

75230 Paris Cedex 05, France

1. J. H. BRAMBLE \& M. ZLÁMAL, "Triangular elements in the finite element method," Math. Comp., v. 24, 1970, pp. 809-820.

2. P. G. CIARLET, "Sur l'élément de Clough et Tocher," Rev. Française Automat. Informat. Recherche Opérationnelle Sér. Rouge Anal. Numér. R-2, 1974, pp. 19-27.

3. P. G. CIARLET, Numerical Analysis of the Finite Element Method, Presses de 1'Université de Montréal, Montréal, 1976.

4. P. G. CIARLET, The Finite Element Method for Elliptic Problems, North-Holland, Amsterdam, 1977.

5. P. G. CIARLET \& P.-A. RAVIART, "General Lagrange and Hermite interpolation in $R^{n}$ with applications to finite element methods," Arch. Rational Mech. Anal., v. 46, 1972, pp. 177199.

6. R. W. CLOUGH \& J. L. TOCHER, "Finite element stiffness matrices for analysis of plates in bending," in Proc. Conf. on Matrix Methods in Structural Mechanics, Wright-Patterson A.F.B., Ohio, 1965.

7. J. NEČAS, Les Méthodes Directes en Théorie des Equations Elliptiques, Masson, Paris, 1967.

8. P. PERCELL, "On cubic and quartic Clough-Tocher finite elements," SIAM J. Numer. Anal., v. 13, 1976, pp. 100-103.

9. P.-A. RAVIART, Méthode des Eléments Finis, Lecture Notes (D.E.A. Analyse Numérique), Laboratoire d'Analyse Numérique, Université Pierre et Marie Curie (Paris VI), 1972.

10. A. ŽENIŠ̌EK, "Interpolation polynomials on the triangle," Numer. Math., v. 15, 1970, pp. 283-296.

11. A. ŽENIŠ́EK, "A general theorem on triangular finite $C^{(m)}$-elements," Rev. Française Automat. Informat. Recherche Opérationnelle Sér. Rouge Anal. Numér. R-2, 1974, pp. 119-127.

12. O. C. ZIEN KIEWICZ, The Finite Element Method in Engineering Science, McGrawHill, London, 1971.

13. M. ZLÁMAL, "On the finite element method," Numer. Math., v. 12, 1968, pp. 394409. 